\title{
Casimir energy in a bounded Gross-Neveu model
}

\author{
F. Escalante and J.C. Rojas \\ Departamento de Física, Universidad Católica del Norte, \\ Antofagasta, 1240000, Chile. \\ e-mail: fescalante@ucn.cl; jurojas@ucn.cl
}

Received 24 April 2018; accepted 19 August 2018

We study the Casimir energy and forces associated with the vacuum of the massless Gross-Neveu (GN) model in a finite spatial dimension for different boundary conditions. The standard solution given by the Hartree-Fock method is considered using the generalized method of the zeta function, with the aim of studying the dynamic generation of mass and the associated beta function. It is found that the beta function does not depend on the boundary conditions. Then, considering several boundary conditions, the corresponding Casimir energies and forces were obtained. We obtain that the nature of the forces depends as much on the type of contour condition as on the magnitude of the space.

Keywords: Renormalization; Casimir effect; Gross-Neveu; zeta function.

PACS: 11.10.Kk; 11.10.Jj; 11.10.Gh

\section{Introduction}

The Gross-Neveu (GN) model describes a system of massless fermions with self-interacting fermions which generates a dynamical mass. The GN model was born as a toy model of Quantum Chromodynamics (QCD) [1]. At first, it was studied using semiclassical functional methods, which is rigorously justified in the large $\mathrm{N}$ limit. In the traditional context, the GN model has shown a rich phase structure, in particular, there we have chiral symmetry breaking. Which, at first sight, it could not be allowed in 1+1 dimensions due to infrared fluctuations, unless it is invoked the large $\mathrm{N}$ limit [2]. Despite its simplicity, it keeps many exciting features, such as discrete chiral symmetry, dynamical mass generation, and asymptotic freedom.

Curiously, this model has also application in condensed matter physics, where it describes the conductivity in some polymers. In particular, it can be mentioned the case of transpolyacetylene, which, in a simplified continuous model, is described by the symmetric GN model [3]. Besides, the massive GN model has a condensed matter analog in the modelization of polymers with non-degenerate ground states [4].

The standard approach to the GN model leads to an homogeneous condensate solution, i.e., independent of the space coordinates. After several years, it was realized that there are crystal solutions of the model, giving a rich interpretation in the realm of condensed matter physics [5]. The spatial dimension could be constrained to a finite size to consider Casimir type forces. Without having in mind any particular model, we asked ourselves to consider many boundary conditions (BC's) to simulate a broad variety of possible physical scenarios. Those are periodic, antiperiodic and confining BC's.

Having a limited spatial extension leads us to consider the Casimir energy associated with such spaces. We found that the Casimir energies and forces are sensitive to the BC's and parameters involved. Using the generic name of "universe" for the spatial dimension we identified the stable, metastable, attractive or repulsive regimes, depending on the parameters and BC's we use

There is previous work in the direction we propose. In particular, in [6], it was computed the Casimir energy with MIT Bag Model boundary condition, obtaining the behavior of the Casimir energy as a function of the distance between the points and the mass of the fermionic field. On the other side, the renormalization issue of the GN model was addressed in [7], using the worldline Montecarlo approach.

In our study, we shall concentrate on the homogeneous solutions of the GN model for a finite space of fixed size $L$. We are interested in the behaviour of physical parameters for different boundary conditions (BC's). The boundary conditions considered are periodic, antiperiodic and two types of confining conditions.

The HF approximation implies the use of a large momentum cutoff. Since we shall deal with systems of finite spatial size, the momentum integrals must be replaced by summation on discrete modes, meaning that the natural regularization to be used is the zeta regularization technique [8].

In this work, we first ask about the ultraviolet dependence of the physical parameters on the BC's, considering the GN model at zero bare mass $\left(m_{0}=0\right)$ where temperature and chemical potential are not considered. We assume that the spatial length $L$ is a fixed parameter, so, if the physical mass is independent of the cutoff, it implies that the beta function does not depend on the BC's. There appears an arbitrary mass scale and the functional dependency of the dynamical mass depends on the BC's.

The second step in our work is to study the Casimir energy and force due to the quantum fluctuation of the effective free system that arises from the HF approximation. We consider the non-dimensional parameter $\mu=m L$, since the value of $m$ is fixed by ultraviolet considerations, the variation of $\mu$ is equivalent to the variation of $L$. We find that the value of energy and Force are sensitive to the BC's. In particular, the signature of the energy clearly differs in the small size 
limit, but it is universally positive for infinite size limit. On the other hand, the force is also sensitive to the BC's, implying situations where the forces are such that they compress or expand our space depending on the BC's used. There is also a universal metastable point where the force becomes zero independently of the BC's used. For the large $L$ limit, the force becomes negative for any BC's considered.

\section{The Gross-Neveu Model}

The Gross-Neveu Lagrangian is given by

$$
\begin{aligned}
\mathcal{L}_{G N} & =\bar{\psi}^{i} i \gamma^{\mu} \partial_{\mu} \psi^{i}+\frac{1}{2} g^{2}\left\{\left(\bar{\psi}^{i} \psi^{i}\right)^{2}-\lambda\left(\bar{\psi}^{i} \gamma_{5} \psi^{i}\right)^{2}\right\} \\
& -m_{0} \bar{\psi}^{i} \psi^{i},
\end{aligned}
$$

where $i$ runs from 1 to $N$. For the sake of simplicity, from now, we suppress the index $i$. The Euler Lagrange equation from (1) is given by

$$
i \gamma^{\mu} \partial_{\mu} \psi+g^{2}\left\{\bar{\psi} \psi-\lambda\left(\bar{\psi} \gamma_{5} \psi\right) \gamma_{5}\right\} \psi-m_{0} \psi=0
$$

In the framework of Hartree-Fock relativistic approximation, it is assumed the expectation value $\left\langle\bar{\psi} \gamma_{5} \psi\right\rangle=0$ and $\langle\bar{\psi} \psi\rangle=N \rho$.

It was introduced a finite mass in order to consider a general expression and using the convention

$$
\begin{array}{ll}
\gamma^{0} & =\left(\begin{array}{cc}
0 & -1 \\
-1 & 0
\end{array}\right), \quad \gamma^{1}=\left(\begin{array}{cc}
i & 0 \\
0 & -i
\end{array}\right), \\
\gamma^{5} & =\left(\begin{array}{cc}
0 & i \\
-i & 0
\end{array}\right),
\end{array}
$$

we end up with the expression

$$
\left(i \gamma^{\mu} \partial_{\mu}-m\right) \psi(x)=0,
$$

where $m=m_{0}-g^{2} N \rho$ and $\rho=\langle\bar{\psi} \psi\rangle / N$.

From (4) we obtain a free Dirac equation

$$
i \frac{\partial \psi}{\partial t}=H \psi=\left(\begin{array}{cc}
0 & -i \\
i & 0
\end{array}\right)\left(-i \partial_{x}\right) \psi-m\left(\begin{array}{ll}
0 & 1 \\
1 & 0
\end{array}\right) \psi .
$$

In order to obtain a stationary solution, we use the usual decomposition

$$
\psi(x)=\mathrm{e}^{-i \lambda_{n} t}\left(\begin{array}{c}
\phi(x) \\
\chi(x)
\end{array}\right) .
$$

Making the redefinition of the fields

$$
f=\chi+\phi, g=\chi-\phi,
$$

we obtain a general solution

$$
\begin{aligned}
& f(x)=\frac{\alpha}{\sqrt{\lambda_{n}+m}} \cos (\Omega x)-\frac{\beta}{\sqrt{\lambda_{n}+m}} \sin (\Omega x), \\
& g(x)=\frac{\alpha}{\sqrt{\lambda_{n}-m}} \sin (\Omega x)+\frac{\beta}{\sqrt{\lambda_{n}-m}} \cos (\Omega x),
\end{aligned}
$$

where $\Omega=\sqrt{\lambda_{n}^{2}-m^{2}}$ and the constants $\alpha$ and $\beta$ are not independent since they are determined by the boundary conditions.

\section{Hartree-Fock for different boundary condi- tions}

Following the standard procedure [9], it is possible to compute the negative energy in an infinite space taking the value of $m$ as a parameter to be determined

$$
\frac{\mathcal{E}}{N}=-2 \int_{|k| \leq \Lambda} \frac{d k}{2 \pi} \sqrt{m^{2}+k_{n}^{2}}+\frac{m^{2}}{2 G},
$$

where $G=N g^{2}$ and $\Lambda$ is a momentum cut off.

Since we have a finite spatial size, the wave number $k$ is discretized

$$
k_{n}=(2 \pi n+\phi) / r L,
$$

implying

$$
\int d k /(2 \pi) \rightarrow \frac{1}{r L} \sum
$$

where $r$ is a number which depends on boundary conditions. So, we have

$$
\frac{\mathcal{E}}{N}=-\frac{2}{r L} \sum_{n}\left(m^{2}+k_{n}^{2}\right)^{1 / 2}+\frac{m^{2}}{2 G}
$$

Dealing with the summation implies a treatment of Epstein zeta function

$$
\zeta_{E}(s ; a, b)=\sum_{n=-\infty}^{\infty}\left(a^{2}+(n+b)^{2}\right)^{-s}
$$

using properties of gamma function and by means of Jacobi inverse summation formulae [8], we have

$$
\begin{aligned}
\zeta_{E}(s ; a, b)= & \frac{\sqrt{\pi}}{\Gamma(s)}\left[\int_{0}^{\infty} t^{s-3 / 2} e^{-t a^{2}} d t+2 \sum_{n=1}^{\infty} \cos (2 \pi b n)\right. \\
& \left.\times \int_{0}^{\infty} t^{s-3 / 2} e^{-t a^{2}-\pi^{2} n^{2} / t} d t\right]
\end{aligned}
$$

From the above integral, we can recognize a gamma function and a second kind Bessel function [10], leading us to the general expression

$$
\begin{aligned}
\zeta_{E}(s ; a, b) & =\frac{\sqrt{\pi}}{\Gamma(s)}\left[a^{-2 s+1} \Gamma\left(s-\frac{1}{2}\right)+4 \sum_{n=1}^{\infty} \cos (2 \pi b n)\right. \\
& \left.\times\left(\frac{\pi n}{a}\right)^{\left(s-\frac{1}{2}\right)} K_{s-\frac{1}{2}}(2 \pi a n)\right]
\end{aligned}
$$


We are interested in the value $s=-1 / 2$, but there is a singularity in such point, so we can isolate that term by meaning of a series expasion in term of $s=-1 / 2+\epsilon$, when $\epsilon \rightarrow 0$, giving us the expression for the Epstein zeta function

$$
\begin{aligned}
\zeta_{E}(\epsilon ; a, b) & =\frac{a^{2}}{2 \epsilon}-\frac{a^{2}}{2}-a^{2} \ln \left(\frac{a}{2}\right) \\
& -\frac{2 a}{\pi} \sum_{n=1}^{\infty} \frac{\cos (2 \pi b n)}{n} K_{1}(2 \pi a n) .
\end{aligned}
$$

where we use tha fact that $K_{n}(x)=K_{-n}(x)$. Note that there is a diverging term, which can be seen as an ultraviolet cutoff. Since the power $1 / 2$ in the summation is replaced by a term $1 / 2-\epsilon$, it appears a mass scale $\eta$ by means of the regularization procedure [8].

We have the following momentum decomposition for the BC's to be considered:

Periodic $\quad k_{n}=2 \pi n / L \quad n \in(-\infty, \infty)$,

Antiperiodic $k_{n}=(2 n+1) \pi / L \quad n \in(-\infty, \infty)$,

Zero current i) $\quad k_{n}=(2 n) \pi / 2 L \quad n \in(-\infty, \infty)$,

Zero current ii) $\quad k_{n}=(2 n+1) \pi / 2 L \quad n \in(-\infty, \infty)$.

We note that the imposed boundary conditions over $k_{n}$ have the general structure

$$
k_{n}^{2}=\frac{(2 \pi n+\phi)^{2}}{r^{2} L^{2}} .
$$

Introducing the parameter of mass $\eta$ and rewriting the energy density we have

$$
\begin{aligned}
\frac{L^{2} \mathcal{E}}{N} & =-\frac{2 L}{r} \eta^{2 s+1}\left(\frac{2 \pi}{r L}\right)^{-2 s} \\
& \times \sum_{n=-\infty}^{\infty}\left(\frac{\mu^{2} r^{2}}{4 \pi^{2}}+\left(n+\frac{\phi}{2 \pi}\right)^{2}\right)^{-s}+\frac{\mu^{2}}{2 G} .
\end{aligned}
$$

As we see, the summation term can be expressed as an Epstein zeta function, so we have

$$
\begin{aligned}
\frac{\mathcal{E}}{N} & =-\frac{\mu^{2}}{2 \pi \epsilon L^{2}}+\frac{\mu^{2}}{2 \pi L^{2}}-\frac{\mu^{2}}{\pi L^{2}} \ln \frac{2 \tilde{\eta}}{\mu} \\
& +\frac{4 \mu}{\pi r L^{2}} \sum_{n=1}^{\infty} \frac{\cos \phi n}{n} K_{1}(\mu r n)+\frac{\mu^{2}}{2 G L^{2}} .
\end{aligned}
$$

Minimizing the energy density respect to $\mu$ we can obtain a dimensionless expression

$$
\begin{aligned}
\frac{L^{2}}{N \mu} \frac{\partial \mathcal{E}}{\partial \mu} & \equiv \mathcal{X}_{\mu}=-\frac{1}{\pi \epsilon}+\frac{2}{\pi}-\frac{2}{\pi} \ln \left(\frac{2 \tilde{\eta}}{\mu}\right) \\
& -\frac{4}{\pi} \sum_{n=1} \cos (\phi n) K_{0}(\mu r n)+\frac{1}{G},
\end{aligned}
$$

as $\mathcal{X}_{\mu}=0$, the coupling parameter $G$ is given by

$$
\begin{aligned}
G & =\pi\left\{\frac{1}{\epsilon}-2+2 \ln \left(\frac{2 \tilde{\eta}}{\mu}\right)\right. \\
& \left.+\frac{4}{\pi} \sum_{n=1}^{\infty} \cos (\phi n) K_{0}(\mu r n)\right\}^{-1} .
\end{aligned}
$$

For the four considered BC's, we obtained the following expressions for the energy density:

- Periodic BC

$$
\begin{aligned}
\frac{\mathcal{E}^{P}}{N} & =-\frac{\mu^{2}}{2 \pi \epsilon L^{2}}+\frac{\mu^{2}}{2 \pi L^{2}}-\frac{\mu^{2}}{\pi L^{2}} \ln \left(\frac{2 \tilde{\eta}}{\mu}\right) \\
& +\frac{4 \mu}{\pi L^{2}} \sum_{n=1}^{\infty} \frac{K_{1}(\mu n)}{n}+\frac{\mu^{2}}{2 G L^{2}} .
\end{aligned}
$$

- Anti periodic BC

$$
\begin{aligned}
\frac{\mathcal{E}^{A P}}{N} & =-\frac{\mu^{2}}{2 \pi \epsilon L^{2}}+\frac{\mu^{2}}{2 \pi L^{2}}-\frac{\mu^{2}}{\pi L^{2}} \ln \left(\frac{2 \tilde{\eta}}{\mu}\right) \\
& +\frac{4 \mu}{\pi L^{2}}\left\{\sum_{n=1}^{\infty} \frac{K_{1}(2 \mu n)}{n}-\sum_{n=1}^{\infty} \frac{K_{1}(\mu n)}{n}\right\} \\
& +\frac{\mu^{2}}{2 G L^{2}} .
\end{aligned}
$$

- Zero current BC

$$
\begin{aligned}
\frac{\mathcal{E}^{i}}{N} & =-\frac{\mu^{2}}{2 \pi \epsilon L^{2}}+\frac{\mu^{2}}{2 \pi L^{2}}-\frac{\mu^{2}}{\pi L^{2}} \ln \left(\frac{2 \tilde{\eta}}{\mu}\right) \\
& +\frac{2 \mu}{\pi L^{2}} \sum_{n=1}^{\infty} \frac{K_{1}(2 \mu n)}{n}+\frac{\mu^{2}}{2 G L^{2}}, \\
\frac{\mathcal{E}^{i i}}{N} & =-\frac{\mu^{2}}{2 \pi \epsilon L^{2}}+\frac{\mu^{2}}{2 \pi L^{2}}-\frac{\mu^{2}}{\pi L^{2}} \ln \left(\frac{2 \tilde{\eta}}{\mu}\right) \\
& +\frac{2 \mu}{\pi L^{2}} \sum_{n=1}^{\infty}(-1)^{n} \frac{K_{1}(2 \mu n)}{n}+\frac{\mu^{2}}{2 G L^{2}} .
\end{aligned}
$$

where it was introduced the non-dimensional variables $\mu=$ $m L$ and $\tilde{\eta}=\eta L$.

In the following step, we minimize the energy densities with respect to $\mu$. Then, we use (21) and obtain for each BC an expression for $G$, which can be resumed to the form

$$
G=\pi\left\{\frac{1}{\epsilon}-2+2 \ln \left(\frac{2 \tilde{\eta}}{\mu}\right)+\text { finite terms }\right\}^{-1},
$$

where $\epsilon=s+1 / 2$ goes to zero and must be considered as the ultraviolet cut-off. On the other side, the finite terms depends on the BC's.

For finite $\epsilon$, it gives the impression that the running of $\mathrm{G}$ should depend on the BC's. But, if we fix the value of $G$ in an arbitrary scale, the limit $\epsilon \rightarrow 0$ is universal, independent of any $\mathrm{BC}$. 


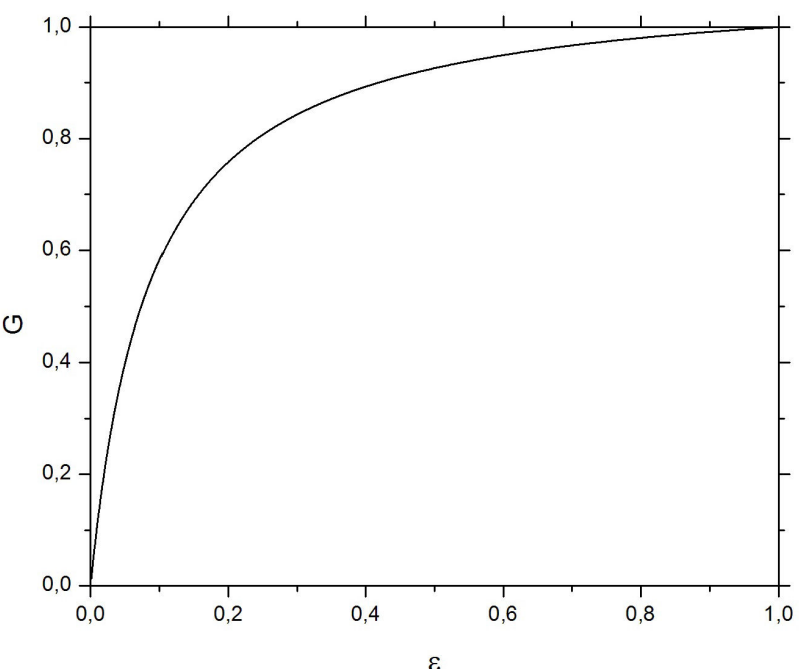

FIGURE 1. The running of $G$, for different BC's fixing the parameters in order to have $G=1$ for $\epsilon=1$.

We observe from the general relation (21), that there is dependency of the constants $\mu$ and $\tilde{\eta}$ for each BC through the transcendental equation:

$$
2 \ln \left(\frac{2 \tilde{\eta}}{\mu}\right)+\frac{4}{\pi} \sum_{n=1}^{\infty} \cos (\phi n) K_{0}(\mu r n)=C,
$$

being $C$ an arbitrary constant.

Considering the traditional point of view where the physical $\mu$ must be independent of the cutt-off $\epsilon$ we have a renormalization group equation

$$
\epsilon^{2} \frac{d \mu}{d \epsilon}=\epsilon^{2} \frac{\partial \mu}{\partial \epsilon}+\epsilon^{2} \frac{\partial G}{\partial \epsilon} \frac{\partial \mu}{\partial G}+\epsilon^{2} \frac{\partial \tilde{\eta}}{\partial \epsilon} \frac{\partial \mu}{\partial \tilde{\eta}}=0 .
$$

For any $\mathrm{BC}$, it is computed the beta function

$$
\beta=\epsilon^{2} \frac{d G}{d \epsilon},
$$

meaning an universal behaviour of $G(\epsilon)$ as it is shown in Fig. 1.

\section{Casimir Energy for global boundary condi- tions}

Imposing BC's of the form

$$
\left(\begin{array}{l}
f(x+L) \\
g(x+L)
\end{array}\right)=\mathbb{M}\left(\begin{array}{l}
f(x) \\
g(x)
\end{array}\right)
$$

In Eqs. (8) and (9) we have solutions of the form

$$
\left(\begin{array}{l}
f(x) \\
g(x)
\end{array}\right)=\left(\begin{array}{ll}
f_{1}(x) & f_{2}(x) \\
g_{1}(x) & g_{2}(x)
\end{array}\right)\left(\begin{array}{l}
\alpha \\
\beta
\end{array}\right)
$$

where the values of $\alpha, \beta$ depend on the imposed $\mathrm{BC}$ on the problem. If we define the invertible matrix

$$
\mathbb{H}(x)=\left(\begin{array}{ll}
f_{1}(x) & f_{2}(x) \\
g_{1}(x) & g_{2}(x)
\end{array}\right),
$$

the constants are given by

$$
\left(\begin{array}{l}
\alpha \\
\beta
\end{array}\right)=\mathbb{H}^{-1}(0)\left(\begin{array}{l}
f(0) \\
g(0)
\end{array}\right)
$$

meaning that

$$
\left(\begin{array}{l}
f(x) \\
g(x)
\end{array}\right)=\mathbb{H}(x) \mathbb{H}^{-1}(0)\left(\begin{array}{l}
f(0) \\
g(0)
\end{array}\right) \equiv \mathbb{M}\left(\begin{array}{l}
f(0) \\
g(0)
\end{array}\right)
$$

The $\mathrm{BC}$ can be expressed as

$$
\left(\begin{array}{l}
f(L) \\
g(L)
\end{array}\right)=\mathbb{M}\left(\begin{array}{l}
f(0) \\
g(0)
\end{array}\right)
$$

Then, the eigenvalue condition is given by

$$
\operatorname{det}\left[\mathbb{M}-\mathbb{H}(L) \mathbb{H}^{-1}(0)\right]=0 .
$$

We can include the periodic and anti periodic case by the parametrization

$$
\mathbb{M}=\left(\begin{array}{cc}
\exp (i \phi) & 0 \\
0 & \exp (i \phi)
\end{array}\right) .
$$

From (8) and (9), we have

$$
\mathbb{H}(x)=\left(\begin{array}{cc}
\frac{\cos (\Omega x)}{\sqrt{\lambda_{n}+m}} & -\frac{\sin (\Omega x)}{\sqrt{\lambda_{n}+m}} \\
\frac{\sin (\Omega x)}{\sqrt{\lambda_{n}-m}} & \frac{\cos (\Omega x)}{\sqrt{\lambda_{n}-m}}
\end{array}\right) .
$$

Eq. (35) leads to the condition

$$
\cos \Omega L=\cos \phi \rightarrow \Omega^{2}=\frac{(2 \pi n+\phi)^{2}}{r^{2} L^{2}}, n \in \mathbb{Z} .
$$

Since $\Omega=\sqrt{\lambda^{2}-m^{2}}$, we have

$$
\begin{aligned}
\lambda_{n}^{2} & =m^{2}+\frac{(2 \pi n+\phi)^{2}}{r^{2} L^{2}} \\
& =\frac{4 \pi^{2}}{r^{2} L^{2}}\left[\frac{\mu^{2} r^{2}}{4 \pi^{2}}+\left(n+\frac{\phi}{2 \pi}\right)^{2}\right] .
\end{aligned}
$$

Where $\mu=m L$ and $r$ a parameter which depends of the boundary conditions, so the general expression for the Casimir energy for spinor field is given by

$$
\begin{aligned}
E_{C a s} & =-\frac{1}{2} \sum_{n} \lambda_{n}^{-2 s}=-\lim _{s \rightarrow-1 / 2} \frac{1}{2}\left(\frac{2 \pi}{r L}\right)^{-2 s} \\
& \times \sum_{n=-\infty}^{\infty}\left[\frac{\mu^{2} r^{2}}{4 \pi^{2}}+\left(n+\frac{\phi}{2 \pi}\right)^{2}\right]^{-s} .
\end{aligned}
$$

We can recognize that the summation term in (37) an Epstein zeta function, so we use the representation in (15) in order to obtain the Casimir energy. We redefine the Casimir energy given by

$$
\begin{aligned}
\xi_{\text {Cas }} & \equiv L E_{\text {Cas }}=\frac{\mu^{2} r}{8 \pi}-\frac{\mu^{2} r}{4 \pi} \ln \frac{2 \eta L}{\mu} \\
& +\frac{\mu}{\pi} \sum_{n=1}^{\infty} \frac{\cos \phi n}{n} K_{1}(\mu r n) .
\end{aligned}
$$


The Casimir Force can be obtained by the usual way $F_{C a s}=-d E_{C a s} / d L$, but considering the redefinition given before and $\tilde{\eta} \equiv \eta L$, we have

$$
\begin{aligned}
\mathcal{F}_{\text {Cas }} & =-\frac{r}{8 \pi}+\frac{r}{4 \pi} \ln \frac{2 \tilde{\eta}}{\mu}+\frac{r}{\pi} \sum_{n=1}^{\infty} \cos (\phi n) K_{0}(\mu r n) \\
& +\frac{1}{\mu \pi} \sum_{n=1}^{\infty} \frac{\cos (\phi n)}{n} K_{1}(\mu r n),
\end{aligned}
$$

where $\mathcal{F}_{\text {Cas }}=F / m^{2}$.

\section{Specific boundary conditions}

\subsection{Periodic and Anti Periodic BC's}

The Casimir energies for the periodic and antiperiodic BC's are given by

$$
\begin{aligned}
\xi_{\text {Cas }}^{P} & =\frac{\mu^{2}}{8 \pi}-\frac{\mu^{2}}{4 \pi} \ln \left(\frac{2 \tilde{\eta}}{\mu}\right)+\frac{\mu}{\pi} \sum_{n=1}^{\infty} \frac{1}{n} K_{1}(n \mu), \xi_{\text {Cas }}^{A P} \\
& =\frac{\mu^{2}}{8 \pi}-\frac{\mu^{2}}{4 \pi} \ln \left(\frac{2 \tilde{\eta}}{\mu}\right) \\
& -\frac{\mu}{\pi} \sum_{n=1}^{\infty}\left(\frac{\left.K_{1} \mu n\right)}{n}-\frac{K_{1}(2 \mu n)}{n}\right),
\end{aligned}
$$

and the Casimir forces are

$$
\begin{aligned}
\mathcal{F}_{\text {Cas }}^{P} & =-\frac{1}{8 \pi}+\frac{1}{4 \pi} \ln \left(\frac{2 \tilde{\eta}}{\mu}\right) \\
& +\frac{1}{\pi} \sum_{n=1}^{\infty} K_{0}(\mu n)+\frac{1}{\mu \pi} \sum_{n=1}^{\infty} K_{1}(\mu n) . \\
\mathcal{F}_{\text {Cas }}^{A P} & =-\frac{1}{8 \pi}+\frac{1}{4 \pi} \ln \left(\frac{2 \tilde{\eta}}{\mu}\right) \\
& -\frac{1}{\pi} \sum_{n=1}^{\infty}\left(K_{0}(\mu n)-2 K_{0}(2 \mu n)\right) \\
& -\frac{1}{\mu \pi} \sum_{n=1}^{\infty}\left(\frac{K_{1}(\mu n)}{n}-\frac{K_{1}(2 \mu n)}{n}\right) .
\end{aligned}
$$

\subsection{Zero current BC}

The confining condition is imposing the zero current condition at the borders

$$
i n^{\mu} \bar{\psi} \gamma_{\mu} \psi=\left.0\right|_{x=0, L} .
$$

In terms of components, we have

$$
\begin{gathered}
\psi=\left(\begin{array}{c}
\phi(x) \\
\chi(x)
\end{array}\right) \rightarrow n^{\mu} \bar{\psi} \gamma_{\mu} \psi=\phi(x) \chi(x)^{*}-\phi(x)^{*} \chi(x) . \\
\text { If } \phi(x)=|\phi(x)| \mathrm{e}^{\alpha}, \chi(x)=|\chi(x)| \mathrm{e}^{\beta}, \text { then } \\
\quad i n^{\mu} \bar{\psi} \gamma_{\mu} \psi=2 i|\phi(x)||\chi(x)| \sin (\alpha-\beta)
\end{gathered}
$$

Since $\alpha$ and $\beta$ are constants, we must impose that at the borders one of the fields must be zero, we can consider the following cases:

i) $\chi(0)=0, \chi(L)=0$ or $\phi(0)=0, \phi(L)=0$,

ii) $\phi(0)=0, \chi(L)=0$ or $\chi(0)=0, \phi(L)=0$.

The conditions are

i) $\sin \left(\sqrt{\lambda^{2}-m^{2}} L\right)=0 \rightarrow \lambda_{n}^{2}=m^{2}+\left(\frac{n \pi}{L}\right)^{2}$.

ii) $\sqrt{\frac{\lambda^{2}}{m^{2}}-1} \cos \left(\sqrt{\frac{\lambda^{2}}{m^{2}}-1} \mu\right)-\sin \left(\sqrt{\frac{\lambda^{2}}{m^{2}}-1} \mu\right)=$ 0 , a transcendental equation that for $n \rightarrow \infty$ behaves as $\lambda_{n}^{2}=m^{2}+\left(\frac{(2 n+1) \pi}{2 L}\right)^{2}$.

Following the notation of (36), we have

$$
\begin{aligned}
& \lambda_{n}^{\mathrm{i}, 2}=m^{2}+\frac{n^{2} \pi^{2}}{L^{2}} \rightarrow r=2 \text { and } \phi=0, \\
& \lambda_{n}^{\mathrm{ii}, 2}=m^{2}+\frac{(2 n+1)^{2} \pi^{2}}{(2 L)^{2}} \rightarrow r=2 \text { and } \phi=\pi .
\end{aligned}
$$

According to Ec. (38) with $r=2$, the Casimir energy and the Casimir force for this eigenvalues are given by

$$
\begin{aligned}
\xi_{\text {Cas }}^{i} & =\frac{\mu^{2}}{4 \pi}-\frac{\mu^{2}}{2 \pi} \ln \left(\frac{2 \tilde{\eta}}{\mu}\right)+\frac{\mu}{\pi} \sum_{n=1}^{\infty} \frac{K_{1}(2 \mu n)}{n} \\
\xi_{\text {Cas }}^{i i} & =\frac{\mu^{2}}{4 \pi}-\frac{\mu^{2}}{2 \pi} \ln \left(\frac{2 \tilde{\eta}}{\mu}\right)+\frac{\mu}{\pi} \sum_{n=1}^{\infty}(-1)^{n} \frac{K_{1}(2 \mu n)}{n} \\
\mathcal{F}_{\text {Cas }}^{i} & =-\frac{1}{4 \pi}+\frac{1}{2 \pi} \ln \left(\frac{2 \tilde{\eta}}{\mu}\right)+\frac{2}{\pi} \sum_{n=1}^{\infty} K_{0}(2 \mu n) \\
& -\frac{1}{\mu \pi} \sum_{n=1}^{\infty} \frac{K_{1}(2 \mu n)}{n}, \\
\mathcal{F}_{\text {Cas }}^{i i} & =-\frac{1}{4 \pi}+\frac{1}{2 \pi} \ln \left(\frac{2 \tilde{\eta}}{\mu}\right)+\frac{2}{\pi} \sum_{n=1}^{\infty}(-1)^{n} K_{0}(2 \mu n) \\
& +\frac{1}{\mu \pi} \sum_{n=1}^{\infty}(-1)^{n} \frac{K_{1}(2 \mu n)}{n} .
\end{aligned}
$$

\subsection{Limiting values}

As can be seen from Figs. 2 and 3, the behaviour for small $\mu$ depends on the BC's. In fact, the parameter $\phi$ determines the sign of the force as $\mu$ goes to zero. We are interested in the sign of the force for $\mu \sim 0$, where the force clearly goes to $\pm \infty$. Keeping the leading terms for $\mu \approx 0$ :

$$
\mathcal{F} \approx \frac{1}{\mu^{2} \pi} \sum_{n=1}^{\infty} \frac{\cos (\phi n)}{n^{2}}+\text { constants. }
$$




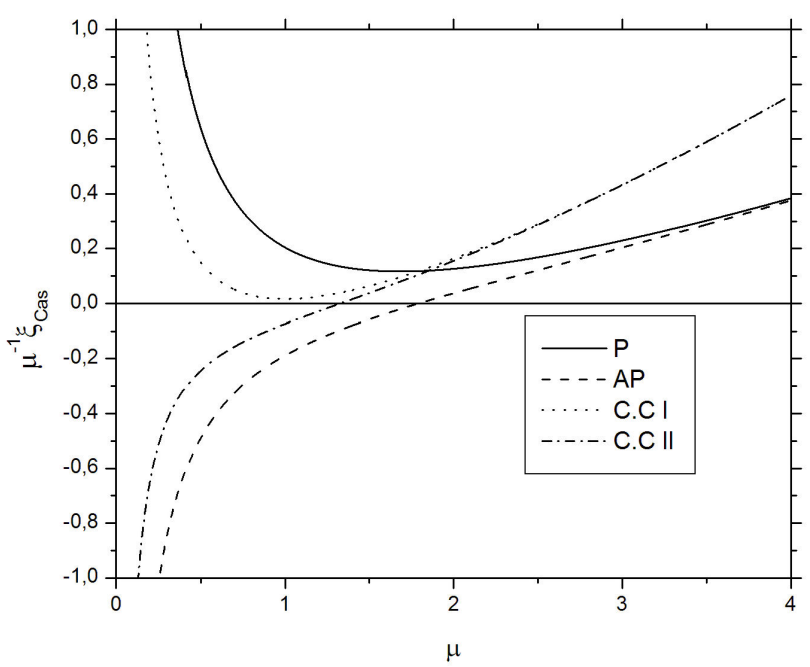

FIGURE 2. The $\mu$ dependency of $\xi$ for different BC's and $\tilde{\eta}=1$. We observe that $\xi^{P}$ and $\xi^{i}$ never reach the zero point energy. We have an asymptotic behaviour coinciding $\xi^{P}$ with $\xi^{A P}$ and $\xi^{i}$ with $\xi^{i i}$.

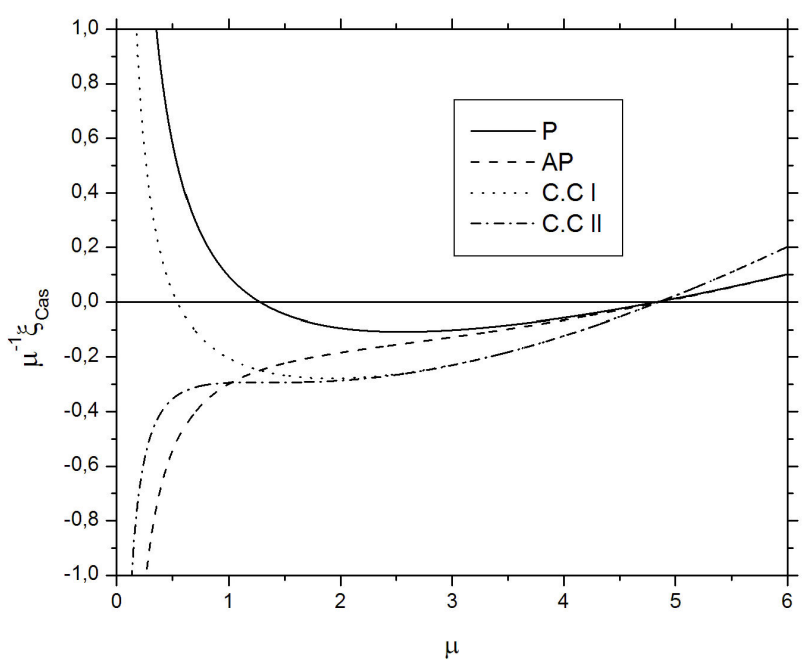

FIGURE 3. Behaviour of $\xi$ for different BC's and $\tilde{\eta}=4$. We obtain that that $\xi^{P}$ and $\xi^{i}$ crosses the zero point energy for a certain region of the parameter $\mu$.

It is more clear to take the derivative to leading order

$$
\frac{d \mathcal{F}}{d \mu}{ }_{\mu \rightarrow 0} \approx-\frac{1}{\mu^{3} \pi}\left[\operatorname{Li}_{2}\left(\mathrm{e}^{i \phi}\right)+\operatorname{Li}_{2}\left(\mathrm{e}^{-i \phi}\right)\right],
$$

where $\operatorname{Li}_{n}(x)$ are Polylogarithm functions (see, for example [11]). Since the positive derivative means a negative force and vice versa. The regime changes for the non physical value of $\phi=\phi^{*} \approx 1.328$, as it is shown in the Fig. 5, notice that $\phi^{*}$ does not depend on $\tilde{\eta}$.

Another curious feature happen with $\mathcal{F}^{A P}$ and $\mathcal{F}^{i i}$. When $\eta$ goes beyond a given value $\tilde{\eta}^{*}=\tilde{\eta}^{*}(\phi)$, the force becomes positive, having an equilibrium points $\mathcal{A}\left(\mathcal{A}^{\prime}\right)$ and a metastable point $\mathcal{B}$, as it is clear from Fig. 6.

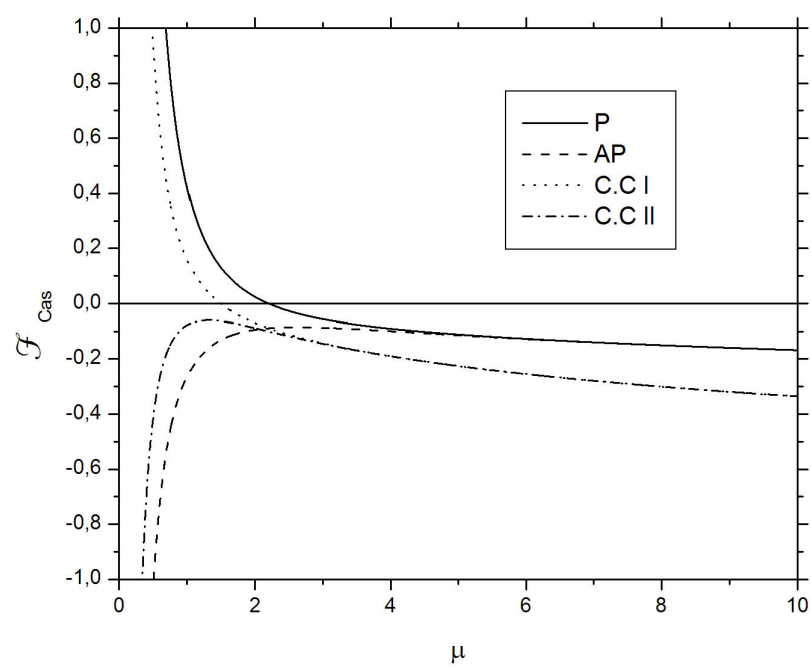

FIGURE 4. The $\mu$ dependency of $\mathcal{F}$ for different BC's and $\eta L=$ $\tilde{\eta}=1$. We can see that $\mathcal{F}^{A P}$ and $\mathcal{F}^{i i}$ are always negative. It is also seen an asymptotic behaviour coinciding $\mathcal{F}^{P}$ with $\mathcal{F}^{A P}$ and $\mathcal{F}^{i}$ with $\mathcal{F}^{i i}$.

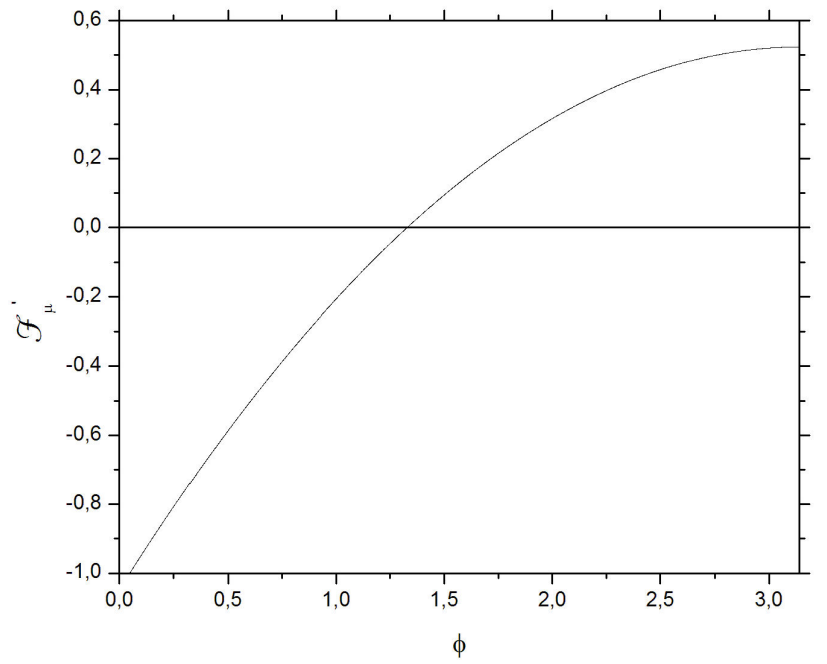

FIGURE 5. Behaviour of the numerator in (50). which indicates the slope of the force when $\mu \approx 0$.

\section{Conclusions and discussion}

The first part of this article focused on the study of the ultraviolet behavior of the GN model for different BC's, using zeta function regularization and assuming a homogeneous solution. We found that the beta function is independent of the type of boundary condition used, and that there appears a mass scale of arbitrary value. The generated dynamical mass should depend on the BC's, if we have no prescription on the arbitrary mass scale.

Later, assuming a homogeneous solution, we studied the Casimir energy and forces for different BC's, if we concentrate on the behavior of $\xi / \mu$ from Figs. 2 and 3, we notice the following features: 


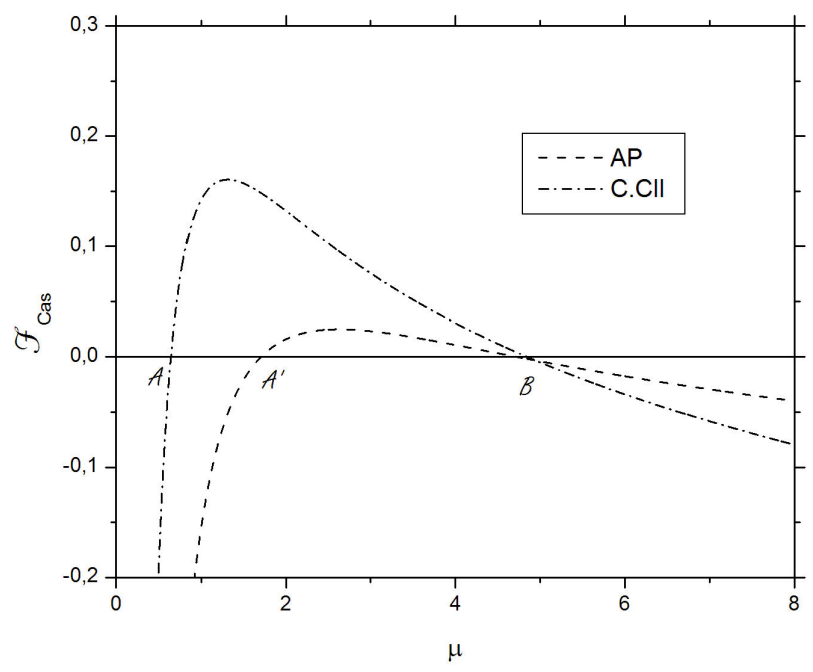

FIgure 6. The values of $\mathcal{F}^{A P}$ and $\mathcal{F}^{i i}$ for $\tilde{\eta}=4$. There it happens that $\mathcal{F}^{A P}$ and $\mathcal{F}^{i i}$ acquire a positive value in region $\mathcal{A}\left(\mathcal{A}^{\prime}\right)$ and becomes zero in the point $\mathcal{B}$.

a) Anti periodic: $\xi / \mu<0$ for $\mu<\mu^{\star}$ and $\xi / \mu>0$ for $\mu>\mu^{\star}$, for any positive value of $\tilde{\eta}$.

b) Periodic: $\xi / \mu$ has a minimum value for a certain value of $\mu=\mu^{\dagger}$, having limiting values of $\xi / \mu \rightarrow \pm \infty$, for $\mu \rightarrow 0$. The sign of the maximum value of $\xi / \mu$, depends on the parameter $\tilde{\eta}$.

c) Confining i: The same qualitative behavior of the periodic case.

d) Confining ii: $\xi / \mu<0$ for $\mu<\tilde{\mu}^{\star}$ and $\xi / \mu>0$ for $\mu>\tilde{\mu}^{\star}$, for any positive value of $\tilde{\eta}$, in a similar fashion as the anti periodic case.

e) We found that there is a common singular value of $\mu$ for $\tilde{\eta} \geq 4$, where $\xi / \mu$ becomes zero.
For the Casimir forces, from Figs. 4 and 6, we conclude that:

1) Anti periodic BC: $\mathcal{F}^{A P} \rightarrow-\infty$ for $\mu \rightarrow 0$ and $\mu \rightarrow \infty$, for any value of $\tilde{\eta}$. It also happen that for $\tilde{\eta} \geq 4, \mathcal{F}^{A P}$ can be positive in a finite range of $\mu$.

2) Periodic: $\mathcal{F}^{P} \rightarrow \infty$ for $\mu \rightarrow 0$ and $\mathcal{F}^{P} \rightarrow-\infty$ for $\mu \rightarrow \infty$

3) Confining i: It has the same qualitative behaviour as the periodic case.

4) Confining ii: It has the same qualitative behaviour as the anti periodic case.

It is shown in Fig. 6 that for $\tilde{\eta} \geq 4$, there is a common point $\mu$ where the Casimir force becomes zero for any boundary condition.

From the above considerations, we conclude that for BC's periodic and confining $i$, there are two regimes of forces, being positive for "small" $\mu$, representing a universe that has an expanding tendency. On the other hand, when $\mu$ is "big", our universe is a shrinking one.

For the antiperiodic and confining ii, there is a more complex situation, since its behavior depends on the value of $\tilde{\eta}$. For $\tilde{\eta} \leq \tilde{\eta}^{*}$, the force is always negative, hence there is an shrinking universe. For $\tilde{\eta} \geq 4$, there is mixed case as it is shown in Fig. 6, there are the points $\mathcal{A}, \mathcal{A}^{\prime}$ and the universal point $B$. Between $\mathcal{A}\left(\mathcal{A}^{\prime}\right)$ and $B$, the force becomes positive. It is also clear that $B$ is an unstable point and the points $\mathcal{A}$, $\mathcal{A}^{\prime}$ are repelling points.

This study suggests that the further natural step is to consider a general relativity study where the spatial dynamics are affected by the quantum fluctuations of the Casimir energy and confirm if the BC's determine the existence of shrinking or expanding low dimensional universes.
1. D. J. Gross and A. Neveu, Phys. Rev. D 10 (1974) 3235.

2. V. Schon and M. Thies, In Shifman, M. (ed.): At the frontier of particle physics, vol. 3 1945-2032. doi:10.1142/97898 128104580041 [hep-th/0008175].

3. M. Thies and K. Urlichs, Phys. Rev. D 72 (2005) 105008.

4. S. A. Brazovskii and N. N. Kirova, JETP Lett. 33 (1981) 4.

5. A. Saxena and A.R. Bischop, Phys. Rev. A 44 (1991) R2251.

6. R. Saghian, M. A. Valuyan, A. Seyedzahedi and S. S. Gousheh, Int. J. Mod. Phys. A 27 (2012) 1250038. doi:10.1142/S0217 751X12500388 [arXiv:1204.3181 [hep-th]].
7. G. Dunne, H. Gies, K. Klingmuller and K. Langfeld, JHEP 0908 (2009) 010. doi:10.1088/1126-6708/2009/08/010

[arXiv:0903.4421 [hep-th]].

8. K. Kirsten, MSRI Publ. 57 (2010) 101.

9. V. Schon and M. Thies, Phys. Rev. D 62 (2000) 096002.

10. I.S. Gradshteyn and I. M. Ryzhik, Table of integrals, series and products (7th Ed.). (New York: Academic Press, 1980).

11. Lewin, Polylogarithms and Associated Functions, (New York, North-Holland Publishing Co., 1981). 